\title{
Approche narratologique à la transmodalisation de Carmen: du récit littéraire à l'opéra
}

\section{Narratological approach to Carmen's transmodalisation: from the literary narrative to the opera}

\author{
MathiLde Robin \\ Universidad de Alicante \\ mr78@alu.ua.es
}

\begin{abstract}
This article presents a study between the Mérimée's novel Carmen and its Bizet's homonym opera from a comparatist perspective, to understand the transmodalisation and mythification process, and also the difference of success between hypotext and hypertext. To that end, it analyses the treatment of the opera's actants in comparison with the novel's actants, but it highlights above all the enhancement brought by the transmodalisation.
\end{abstract}

\section{Key-words}

transmodalisation, comparatism, opera, literature, mythification.

\section{Resumen}

Este artículo presenta un estudio entre la novela Carmen de Mérimée y la ópera de Bizet desde una perspectiva comparatista. Ello permitirá entender el proceso de transmodalización y de mitificación, así como la diferencia de éxito entre hipotexto e hipertexto. Para ello, se analizará el tratamiento de los actantes de la ópera en comparación con los de la novela. Se incidirá sobre todo en el enriquecimiento aportado por la transmodalización.

\section{Palabras clave}

transmodalización, comparatismo, ópera, literatura, mitificación. 


\section{Introduction}

Nous pouvons affirmer que Carmen (1875), l'opéra-comique en quatre actes, composé par Georges Bizet sur le livret de Ludovic Halévy et d'Henri Meilhac, est très certainement l'opéra le plus connu et le plus représenté dans le monde entier. Il en arrive même à faire parfois sombrer dans l'oubli son hypotexte littéraire, la nouvelle de Prosper Mérimée, Carmen (1845). En effet, bien que cette nouvelle littéraire aie toujours été appréciée en France, elle est bien trop souvent restée dans l'ombre de son hypertexte opératique. De plus, son auteur a bien trop souvent été sous-estimé dans le domaine littéraire, malgré ce célèbre mythe moderne basé sur le motif de la femme fatale auquel il a donné naissance. Quant à l'opéra, son succès est indéniable et ses mélodies sont partie intégrante de la culture internationale: très souvent réutilisées dans le domaine de la musique, du cinéma ou de la publicité par exemple, elles font référence au motif de la séduction, ou tout simplement à la culture espagnole: "Fuera de España, decir 'seductora gitana española' es aludir a Carmen; hablar de 'toreros' es evocar la canción de Escamillo. La ópera de Bizet ha llegado a alcanzar un estatus proverbial'" (Jacobs \& Sadie, 1998: 350). En effet, l'image de l'Espagne, et surtout de l'Andalousie, transmise par la nouvelle et d'autant plus par l'opéra, est maintenant devenue le symbole universel de la culture gitane, andalouse et, d'une manière généralisatrice, de la culture espagnole. Ainsi, l'opéra a toujours été admiré en France, malgré une première impression un peu choquante: le mélange de tons comiques avec la tragédie et certains éléments scéniques, comme les femmes débraillées, fumant et crachant sur scène, ou même la mort brutale de Carmen, étaient innovateurs et en quelque sorte provocateurs, car ils transgressaient les règles de la comédie et de la bienséance. À ce propos, Robin May nous indique que la cause de la médiocre réception de la première provenait de "la Opéra Comique, donde simplemente no era costumbre que las óperas terminasen con una muerte violenta, aparte de que a los respetables patrocinadores del teatro no les gustaba, al parecer, ver en escena a gentes del tipo de los amigos de la heroína" (May, 1987: 96). Cependant, malgré une première difficile due au choc culturel avec le type d'audience de l'Opéra Comique, ce chef-d'œuvre musical a vite accroché le public une fois cette première surprise passée.

En revanche, l'image apportée par le mythe de Carmen a été très mal reçue en Espagne, le pays qu'elle est toutefois censée représenter. En effet, le contexte socioculturel n'était guère favorable à cette époque où l'Espagne se sentait de plus en plus méprisée par le reste de l'Europe, dont elle ne partageait ni les idéaux de l'Illustration européenne, ni le Romantisme du XIX ${ }^{\mathrm{e}}$ siècle. Ainsi, les Espagnols s'étaient forgé une attitude défensive envers les préjugés européens, tel que nous l'explique Alberto González Troyano:

La reacción en los cenáculos del pensamiento español ha sido, en general y a lo largo de más de un siglo, contundente: en Mérimée y en su Carmen está, si no todo el origen, sí el germen más sustancial de esa denigrada imagen (que Bizet vino a consolidar) 
de "la España de charanga y pandereta". Con esta fórmula se ha procurado repudiar y exorcizar la visión de una España mala por extranjerizante, artificial y pintoresca (González, 2007: 133).

Ainsi, les intellectuels espagnols et les plus hautes classes sociales espagnoles ne voulaient pas que l'image d'un tel type de femme puisse s'identifier à la femme espagnole, ainsi que les mœurs gitanes soient confondues avec les traditions et la culture espagnoles. Il est vrai que l'assimilation culturelle et l'utilisation du stéréotype sont flagrants pour tout connaisseur de ces différentes cultures, et qu'ils renforcent d'autant plus le stéréotype culturel encore présent actuellement dans les mentalités dans le monde entier. De ce fait, si pour les Espagnols Carmen n'est pas réaliste, pour le public français et européen, c'est une œuvre tout à fait vraisemblable. Son public d'origine, contemporain, bourgeois, illustré et romantique, cherche à s'évader à travers l'exotisme et imagine parfaitement l'Espagne de la façon qu'elle est décrite dans la nouvelle de Mérimée. Le problème survient lors de la réexportation du mythe à son scénario d'origine où il souffre un autre type de lecture, qui, de par l'attitude défensive espagnole, décèle une critique ou même une moquerie de la part d'un étranger.

Somme toute, l'opéra a déclenché un certain scandale auprès des critiques français de l'époque, choqué par cette histoire scabreuse sur la scène de l'Opéra-Comique, ainsi que pour les Espagnols, qui ne s'y sentaient pas du tout identifiés. Mais le succès n'en a pas été freiné, malgré tous les obstacles qui lui ont été posés.

\section{Pour une approche au comparatisme entre musique et littérature}

Nous pouvons donc affirmer que ces deux œuvres françaises font partie intégrante des grands classiques de la littérature et de la musique françaises, et que leur qualité est remarquable. C'est pour cela que nous proposons de faire le rapprochement depuis une perspective comparatiste entre l'hypotexte littéraire et l'hypertexte opératique, ne serait-ce que pour chercher à expliquer la si grande différence de succès entre ces deux œuvres. Dans ce but, nous organiserons cet article de façon à analyser les œuvres de façon parallèle, en partant des informations les plus générales aux plus concrètes. Dans une première partie, nous commencerons par faire une approche théorique de la relation intertextuelle entre la musique et la littérature. Ensuite, nous commenterons certains aspects cruciaux en relation avec les auteurs et le contexte historique, ainsi que la réception internationale des œuvres. Mais, nous nous pencherons surtout sur les choix d'adaptation des librettistes et du compositeur, que ce soit au niveau de la focalisation de la narration, de la trame ou des actants, tout en soulignant l'apport de la musique et de la mise en scène dans cette première réécriture du mythe de Carmen.

À ce propos, nous devons signaler qu'il existe de nombreuses analyses comparatistes entre la nouvelle et les différents hypertextes (dont l'opéra) qui en ont été tirés, mais à notre connaissance, le seul point abordé par les essais dans cette perspective comparatiste reste la 
trame de l'histoire et la notion de fidélité qui est depuis de nombreuses années dépassée par celle de transtextualité. Avant tout, nous devons souligner la grande qualité de deux études analytiques qui ont servis particulièrement d'appui pour cet article: Carmen de Georges Bizet de Blas Cortés et Don Juan, Fígaro, Carmen d'Alberto González Troyano. Afin de compléter ces références bibliographiques remarquables, nous indiquons également les mises en scène de l'opéra sur lesquelles nous nous sommes basé, une sélection due à la grande qualité de leur production: la mise en scène de Franco Zeffirelli à l'Arène de Vérone en 2003, avec Marina Domashenko et Marco Berti; celle de Gianfranco de Bosio au Festival de l'opéra de St. Margarethen de 2005, avec Nadia Krasteva et Aleksandrs Antonenko; celle de Francesca Zambello au Royal Opera House en 2007, avec Anna Caterina Antonacci et Jonas Kaufmann; et celle de Richard Eyre au Metropolitan Opera en 2009, avec Elina Garança et Roberto Alagna.

Pour commencer, nous signalerons que, suivant la théorie de Gérard Genette dans Palympsestes, la relation intertextuelle entre un opéra adapté sur une œuvre littéraire et cette dernière est évidemment une relation d'hypertextualité, pour laquelle nous nous référerons à l'opéra comme l'hypertexte et à la nouvelle de Mérimée comme l'hypotexte'. C'est d'ailleurs le type de relation transtextuelle le plus commun dans les réécritures d'un mythe. De plus, cette hypertextualité se caractérise ici par une transformation de régime sérieux, donnant lieu à une transposition de type transmodalisatrice, c'est-à-dire qu'il s'agit d'un changement de style et non pas de sujet, qui consiste de plus à un changement de domaine artistique: de la littérature à la musique.

Or, à première vue, ces deux arts pourraient sembler ne pas avoir un rapport aussi intéressant et complexe qu'il ne l'est. Effectivement, le premier rapprochement qui nous vient à l'esprit est qu'il s'agit de deux formes artistiques qui ont une incroyable capacité d'expression lyrique, mais dont les formes ne sont pas compatibles ou ressemblantes. Cependant, nous devons faire remarquer que leur origine est la même, et que leur évolution est intrinsèquement liée, comme nous l'affirme Jean-Louis Backès:

Littérature et musique se rencontrent, la parole et le chant sont proches. On dirait que les poètes et musiciens ont d'abord compris les secrets de leur art en méditant sur la

$1 \quad c f$. la définition de l'hypertextualité de Gérard Genette dans Palimpsestes: "J'entends par là toute relation unissant un texte B (que j'appellerai hypertexte) à un texte antérieur A (que j'appellerai, bien sûr, hypotexte) sur lequel il se greffe d'une manière qui n'est pas celle du commentaire." (Genette, 1982: 11-12); et des différents types d'intertextualité: "Par transmodalisation, j'entends donc plus modestement une transformation portant sur ce qu'on appelle, depuis Platon et Aristote, le mode de représentation d'une œuvre de fiction: narratif ou dramatique. Les transformations modales peuvent être a priori de deux sortes: intermodales (passage d'un mode à l'autre) ou intramodales (changement affectant le fonctionnement interne du mode). Cette double distinction nous fournit évidemment quatre variétés, dont deux sont intermodales: passage du narratif au dramatique ou dramatisation, passage inverse du dramatique au narratif ou narrativisation, et deux intramodales: les variations du mode narratif et celles du mode dramatique." (Genette, 1982: 323). Nous appliquons le phénomène de dramatisation à un genre très proche du théâtre, l'opéra, tout en sachant que le composant musical de ce dernier ajoute une complexité à ce type de transmodalisation. 
mélodie; on dirait que de l'unité première, peut-être mythique, tôt rompue, il subsiste des traces: une musique dans la phrase, une voix dans les flûtes et les violons. (Backès, 1994: 22-23)

En fin de compte, ces deux arts s'appuient avant tout sur la voix, sur le don de la parole qui nous permet justement d'être capables d'art: la littérature se base complètement sur la parole, et la musique est née sous forme de poésie chantée, et bien que la musique instrumentale se soit développée sans une forme de parole évidente, elle se base en grande partie sur une imitation du langage. À propos de la relation entre la musique et le langage, Nicolas Ruwet avance:

En principe, il n'y a aucune incompatibilité entre musique et langage, il est toujours possible aux structures musicales de fonctionner [...] sans interférer avec les structures linguistiques, et, si des interférences se produisent, elles ne sont jamais indifférentes: la relation musique-langage est toujours pertinente. (Ruwet, 1972: 53)

En ce qui concerne l'opéra, il semble déjà se rapprocher un peu plus à la littérature qu'une forme musicale plus simple (surtout du théâtre ou de la poésie), grâce à l'utilisation du langage et à son récit narratif. Cependant, la relation entre les deux arts est beaucoup plus complexe que cela, surtout si nous choisissons de comparer une nouvelle, qui est une forme littéraire narrative et non pas poétique ou théâtrale, et un opéra, qui est une forme artistique composée de texte, de musique, mais aussi d'une représentation scénographique. Cette relation est décrite de la façon suivante par Fernando Herrero:

Las relaciones del mundo musical con la literatura, la historia, la poesía y el mito son claras y rotundas: los grandes sentimientos del hombre se expresan hablando o cantando. [...] Así, en la memoria se fijan más esos temas musicales, esa orquestación que los expresa que la diagnosis dialéctica del texto original a través de la potenciación expresiva del canto. [...] es más profunda y duradera que la de la frase o el poema (Herrero, 1983: 169).

Ainsi, nous pouvons affirmer que l'opéra, est l'idéal de l'expression lyrique. En effet, les mots gagnent une signification plus complexe et plus intense grâce à la ligne mélodique et à l'accompagnement orchestral, comme le souligne à son tour David Pujante: "el espectáculo operístico no sólo no quita valencias a los textos dramáticos, sino que los potencia.” (Pujante in Alemany \& Chico, 2012: 472). L'apport de la valeur musicale à un texte écrit est donc incalculable, mais surtout indéniable, de par sa capacité dramatique, sans compter l'apport scénographique dont nous parlerons infra. 


\section{L'avènement d'un nouveau mythe}

La particularité d'un mythe moderne est qu'il apparaît de manière imprévisible, l'auteur n'étant pas conscient de la répercussion postérieure de son œuvre sur les différents arts à travers le monde. Comme nous l'avons mentionné, le mythe de Carmen a commencé par la création de la nouvelle de Prosper Mérimée, et sa première relecture s'est faite à travers de l'opéra de Bizet. Penchons-nous maintenant sur le début de ce processus de mythification.

\subsection{La genèse littéraire de Carmen}

Prosper Mérimée a combiné ses connaissances en archéologie, en anthropologie et en linguistique, ainsi que sa sensibilité littéraire, sa minutie, son esprit critique et son cynisme pour faire la genèse de ce fameux mythe. C'est son travail d'archéologue qui l'amène à visiter pour la première fois l'Espagne, pays dont il est immédiatement fasciné, autant par l'architecture arabisante que par les femmes enivrantes de l'Andalousie. C'est à partir des connaissances acquises durant ses voyages et d'une histoire écoutée de la bouche de la Comtesse de Montijo qu'il a écrit Carmen. De plus, pour alimenter ce personnage de la bohémienne tuée par son amant, il se sert du souvenir d'une jolie jeune femme rencontrée lors de l'un de ses voyages, du nom de Carmencita, et qui semblait être à mi-chemin entre la prostitution et la sorcellerie.

Si nous pouvons souligner une caractéristique de Prosper Mérimée, il s'agit de son souci de peindre fidèlement la couleur locale: ses brèves descriptions possèdent un caractère réaliste et presque documentaire, sans détour à l'heure d'exprimer les sentiments. C'est un auteur qui oscille entre le caractère logique des Lumières, le Romantisme (courant esthétique contemporain à son activité littéraire) et un avant-goût de Réalisme. D'ailleurs, Manuela San Miguel constate que "Mérimée será romántico, pero su romanticismo, fundado en el espíritu del siglo dieciocho ideológico, no será sentimental, lírico, ni cristiano, sino por el contrario, prosaico e intelectual" (San Miguel, 1984: 100). Ce souci de refléter la couleur locale s'apprécie de façon évidente dans la présence des thèmes de culture espagnole et gitane, tels que la superstition et la violence (dans la tauromachie et dans les actions des bandits gitans). À ce sujet, San Miguel ajoute que l'auteur "se siente prendado por el auténtico color local, y su inclinación por las costumbres primitivas y populares le lleva hacia la canaille, siendo arrieros y toreros, a falta de bandoleros auténticos, sus más frecuentes compañías" (San Miguel, 1984: 35). Ainsi, toutes les situations narratives de la nouvelle se basent sur des peintures de la vie quotidienne, que ce soient les traditions espagnoles ou encore les habitudes des bandits gitans.

Par rapport à la superstition, nous pouvons indiquer que Mérimée se montre très attiré par les sciences occultes, et il avoue même les avoir étudiées malgré son athéisme prononcé, 
ce qui se reflète à travers l'aspect fantastique de certains épisodes de l'œuvre. Son anticléricalisme est également constant, laissant claire son opposition au mariage, qui est dépeint comme un échec: dans Carmen, il s'oppose de façon évidente à un type conventionnel de relation amoureuse, et il attribue le pouvoir de séduction de l'héroïne à la sorcellerie, ou bien il la compare au diable de façon répétitive. D'autre part, nous percevons dans la tendance de José à qualifier Carmen de sorcière, de démon ou d'incarnation du diable, une évidente représentation du contexte historique, une référence à un événement qui avait pris lieu juste avant la rédaction de l'œuvre: le dernier autodafé de l'Inquisition au Pays Basque. Ainsi, dans Carmen, nous pouvons voir de nombreuses superstitions à propos de la sorcellerie et des multiples allusions au diable, ainsi que la fleur de cassie qui sert de filtre d'amour.

D'autre part, même si nous avons signalé que Mérimée ne s'identifiait pas à ce mouvement littéraire, le $\mathrm{XIX}^{\mathrm{e}}$ siècle est le siècle du Romantisme, qui a supposé un chavirement des valeurs rationalistes et des mœurs classiques dans toute l'Europe des Lumières. Or, si de nombreux personnages romantiques masculins ont alors surgi, les femmes ont toutefois continué à être trop souvent considérées comme l'objet de séduction des hommes, comme un "trophée de chasse". Il existe donc encore une forte misogynie, surtout dans la littérature espagnole. Il a fallu qu'un Français, Prosper Mérimée, à travers Carmen, fasse sortir la femme, et surtout la femme espagnole, de ce rôle stéréotypé, donnant lieu à un type de femme qui provoquera de nombreuses controverses. En effet, cette femme fatale incarnée par Carmen est aussi la représentation de la femme de la classe la plus basse de l'échelle sociale espagnole, puisqu'elle est gitane, en opposition à ces femmes fatales qui obtiennent leur pouvoir de leur statut social et de leur richesse.

Or, cette ethnie si particulière n'a obtenu de valeur et de considération littéraire qu'avec le Romantisme, qui était attiré par l'exotisme et par la pureté primitive de ses coutumes. Chercher la beauté dans le peuple est une caractéristique de l'esthétique romantique, intensifiée par l'appréciation de l'exotisme du cadre espagnol de Carmen et par l'ésotérisme qui entoure leur culture et qui s'adapte également à l'aspect fantastique qui imprègne souvent les œuvres romantiques. À ce propos, Rouart suggère:

Le romantisme littéraire s'intéresse au fatum suggéré par la bohémienne en haillons. Entre malédiction et bénédiction, le domaine allemand fait triompher la Tsigane comme hérö̈ne mythique, grâce au topos de sa "noirceur" omniprésente comprise en termes de nature, grâce aussi au mystère de son vagabondage millénaire poétisé en errance et à son jeu avec le destin. (Rouart in Brunel, 2002: 1868)

D'autre part, toujours intéressé par la condition humaine, Mérimée s'est penché sur les conséquences de la Révolution industrielle qui s'étendait en Europe et qui a créé une nouvelle classe sociale miséreuse: la classe ouvrière. Comme mentionné plus haut, l'auteur apprécie la compagnie de cette population modeste et y fait clairement allusion dans Carmen, en décrivant le travail des cigarières espagnoles. Le soin de l'auteur dans la recompilation 
d'informations autour de l'actualité et de la couleur locale, et la retranscription de la mentalité espagnole selon son contexte socioculturel sont ainsi remarquables, créant une certaine vraisemblance dans son écrit de fiction.

\subsection{La mythification de Carmen par sa transmodalisation à l'opéra}

Or, comme nous l'avons expliqué supra, si un mythe commence par une première œuvre et un premier auteur, bien entendu, il ne serait pas considéré comme un mythe s'il n'avait pas été repris et recréé par d'autres œuvres et d'autres auteurs a posteriori. En effet, Mérimée n'est pas le seul créateur du mythe de Carmen, car les librettistes et le compositeur de l'opéra ont contribué à la prise d'importance du mythe pour la culture occidentale, et l'on fait entrer dans la culture universelle. En fait, un auteur n'est jamais conscient d'avoir créé un mythe jusqu'à le voir dans la postérité, lorsque le succès de sa réception mène d'autres auteurs à réutiliser ses thèmes: "au demeurant, même si le personnage mythique est dû à un créateur unique, [...] il ne tourne au mythe que grâce à l'accueil étendu et sans cesse renouvelé qui lui est fait par la postérité. Le mythe a toujours un aspect collectif' (Albouy, 1998: 145). Ce sont les réinterprétations d'un récit qui en font un mythe.

D'une part, Carmen se base déjà sur le motif de la femme fatale qui date de l'Antiquité, motif qui a repris son essor à travers ce nouveau mythe du $\mathrm{XIX}^{\mathrm{e}}$ siècle, et atteignant son apothéose pour la modernité socioculturelle. Les réinterprétations (d'abord l'opéra de Bizet, puis les nombreux hypertextes littéraires, musicaux, cinématographiques et picturaux qui en découlent) convertissent alors le récit en mythe et l'enrichissent considérablement, chaque hypertexte lui apportant un nouveau signifié. Lefter nous le dépeint de la manière suivante:

Pierre Albouy, utilise le terme de palingénésie, désignant en grec une renaissance et une métamorphose, pour décrire l'infini renouvellement du mythe, en raison du caractère inépuisable de ses significations symboliques. Chaque réécriture littéraire du mythe ajouterait encore des signifiés à la référence empruntée, et créerait de nouveaux mythes en retour (Lefter, 2008: 8)

Nous pouvons donc affirmer que Carmen souffre d'un processus de mythification trente ans après sa création, tout cela grâce à la transmodalisation à l'opéra faite par Georges Bizet et ses librettistes Ludovic Halévy et Henri Meilhac. George Bizet leur a commandé ce livret adapté à partir de la nouvelle de Prosper Mérimée, après avoir constaté le succès des livrets qu'ils avaient déjà créés ensemble pour les opéras d'Offenbach. En effet, les deux librettistes et dramaturges formaient une association talentueuse, combinant les connaissances littéraires et culturelles de Halévy et le côté loufoque et fantaisiste de Meilhac. Quant au compositeur, Bizet, il apporte l'exotisme dont la globalité de son œuvre est imprégnée, comme le montrent plusieurs de ses opéras (Les pêcheurs de perles, La jeune fille de Perth et Djamileh). Cependant, au contraire de Mérimée, Bizet n'a jamais visité l'Espagne, ce qui 
a sustenté beaucoup de critiques par rapport à son opéra, surtout de la part de ses contemporains espagnols, qui ne se sentaient pas identifiés par cette œuvre. Néanmoins, son intuition et son imagination lui ont permis de mettre en scène Carmen avec une sensibilité digne de son créateur. Ensemble, ils ont pris le soin de refléter cette couleur locale en parsemant le livret d'indications scéniques qui transmettent certains détails fournis par Mérimée dans sa narration et en exigeant toujours une certaine fidélité dans les différentes mises en scène de l'opéra.

D'autre part, le fait que ce soit une tragédie avec des éléments comiques donne sa force à l'œuvre en représentant le mélange de tons de la réalité. En effet, l'opéra-comique, selon la définition française ${ }^{2}$, était un genre d'opéra particulier qui intégrait des dialogues parlés et non pas chantés en récitatifs. Ainsi, Carmen est un opéra-comique tragique, qui s'appuie toutefois sur des éléments comiques et ironiques, et sur une légèreté entraînante, malgré la fatalité latente. D’après Cortés: “Bizet aprovechó en su beneficio los caracteres ligeros, y aun cómicos, del género. Si Carmen es una tragedia, sentimos más su fuerza por la base realista que le proporcionan esas partes ligeras de la obra" (Cortés, 1986: 27). Il est possible que Bizet transgresse les règles de l'opéra-comique en mélangeant les tons dans un souci de réalisme, suivant la ligne de la nouvelle de Mérimée qui pioche dans toutes les tonalités littéraires pour donner l'effet voulu de vraisemblance. De cette manière, nous pouvons voir dans la nouvelle de Mérimée, mais aussi dans l'opéra, des personnages qui fument, crachent, lisent le tarot, dansent ou chantonnent de façon spontanée, par exemple.

Nonobstant, pour en revenir à la critique faite à l'opéra de Bizet, c'est surtout de son wagnérisme qu'il est accusé par ses contemporains, ce qui, à cette époque, voulait dire que l'on considérait une composition musicale beaucoup trop osée et innovatrice, de telle façon qu'elle en perdait le sens musical - d'autant plus que le contexte musical français se défendait alors de toute influence musicale allemande à cause des tensions entre les deux pays. À ce sujet, Cortes nous explique que: "Las acusaciones repetidas contra su música fueron siempre las mismas [...]: dureza armónica, orquestación osada y wagnerismo flagrante y de pésimo gusto" (Cortes, 1986: 12). Pourtant, la musicalité de Bizet est riche en couleurs et pleine d'énergie, composée avec brio grâce à la clarté donnée par des articulations subtiles, mais aussi grâce à la limpidité du son et à son élégance. C'est avant tout la brillante association de couleurs et de tons qui rend ses mélodies riches et entraînantes. D'ailleurs, Bizet est persuadé d'avoir trouvé sa voie avec Carmen, mais il n'a malheureusement pas pu la développer ni profiter de son succès à cause de sa mort prématurée, trois mois après la première de l'opéra.

2 Comme nous l'expliquent Jacobs et Sadie dans la Guía de la ópera: “La obra fue una ‘ópera cómica' en el sentido francés de la expresión; es decir, una ópera en la que se utilizaba el diálogo hablado pues el término 'ópera cómica' no implica necesariamente la comicidad de la trama.” (Jacobs \& Sadie, 1998: 326) 


\section{Le renforcement scénographique et musical de la trame et des actants à travers la transmodalisation de la nouvelle}

Ainsi, nous avons pu apercevoir une certaine ressemblance entre la tonalité littéraire et le genre lyrique, et entre le réalisme et la vraisemblance qu'inspirent les deux œuvres. Les auteurs étaient portés par le même désir de peindre une couleur locale la plus proche possible de la réalité. Bizet et ses librettistes ont donc été fidèles dans ce sens à leur hypotexte littéraire, mais qu'on-t-il modifié dans leur processus de transmodalisation? Nous verrons qu'il s'agit non seulement d'une contrainte, mais aussi d'un enrichissement pour le mythe.

\subsection{Contrainte vs enrichissement du processus transmodalisateur}

Malgré les nombreuses différences entre la trame de l'hypotexte et celle de l'hypertexte, nous pouvons remarquer que les traits essentiels du mythe sont bel et bien présents dans l'hypertexte. Par exemple, en lisant le livret de l'opéra, il nous résulte évident la reprise directe de phrases ou de dialogues entiers de la nouvelle. Cependant, la forme scénique de l'opéra, si différente à la forme narrative littéraire, a restreint énormément la liberté d'adaptation des librettistes, et a donc limité la transmodalisation de la nouvelle. Ainsi, nous pouvons noter plusieurs grandes différences entre les deux récits, comme le début du livret qu'ils ont choisi de faire in media res, le choix de personnages et d'actions différents (choix justifiés par des raisons pratiques et éthiques), ou la contrainte d'éliminer les parties non-narratives de l'hypotexte. Ainsi, l'opéra n'est en réalité basé que sur le chapitre trois de la nouvelle, qui contient toute l'action narrative, laissant totalement de côté les autres chapitres plus dissertatifs. Les librettistes et le compositeur ont su diviser habilement ce long chapitre en quatre actes de longueur et d'importance égales. Ces choix et/ou contraintes leur ont permis de rendre l'opéra beaucoup plus attrayant et compréhensible pour le public.

En relation aux raisons pratiques et éthiques auxquelles nous faisions référence, nous devons souligner le fait que les librettistes se sont vus obligés d'adapter la nouvelle non pas seulement à un genre et un art différent (changements de la trame aux raisons pratiques), mais aussi au public de l'Opéra-comique de Paris qui ne s'attend pas à une mise-en-scène aussi crue que la nouvelle (changements de la trame aux raisons éthiques, c'est-à-dire qu'il s'agit d'une adaptation due à la règle de bienséance). En effet, nous devons souligner la liberté de la littérature face aux règles de bienséance encore très présentes au théâtre: il est évident que lire un acte violent ou même la mort d'un personnage n'est pas aussi choquant que de voir sa représentation sur scène. Par conséquent, ces raisons éthiques ont contraint les librettistes à supprimer avant tout la plupart des crimes commis par les brigands bohémiens et à diminuer le nombre d'amants de Carmen assassinés par José. Toutefois, ils ont choisi de garder la violence de la mort de Carmen intacte, avec deux coups de poignards soudains -ain- 
si que d'autres actions provocatrices, comme fumer ou cracher sur scène. Quant aux grands changements de trame et de personnages, nous les aborderons dans les parties suivantes de l'article.

Mais avant cela, nous nous arrêterons sur l'enrichissement que la transmodalisation a apporté au mythe malgré les contraintes décrites ci-dessus. Effectivement, certains aspects de la nouvelle de Mérimée sont renforcés par l'opéra, de par les caractéristiques scénographiques (costumes et décors généralement décrits par les didascalies du livret) et musicales (paroles et mélodies). Ces caractéristiques ont un effet important sur les sensations du public et qu'elles renforcent le message transmis par les paroles. L'utilisation du leitmotiv -qui a valu à Bizet les accusations de wagnérisme- est particulièrement intéressante en ce qui concerne la caractérisation de chacun des actants. La variété des formes musicales est frappante (vingt-sept numéros composés de solos, de duos, d'un trio, d'un quintet et d'un sextet, ainsi que de nombreuses interventions des chœurs) et reflète la réalité multiple du monde. À ce propos, Blas Cortés affirme que l'opéra “muestra una curiosa sucesión entre partes solistas, intervenciones del coro, melodrama ${ }^{3}$ y diálogo hablado. El conjunto de la obra nos aparece sumamente contrastado y abigarrado" (Cortés, 1986: 28). La présence presque constante des chœurs représente la foule qui commente les actions des personnages. Par exemple, le chœur final caractérise bien le désordre et le tapage de la foule avant la corrida du dernier acte. Ils remplacent en quelque sorte le narrateur en commentant les événements sur différents tons. Par exemple, ils font la pantomime d'un trio amoureux entre les passants de la place dans le premier acte, ce qui annonce le futur trio amoureux entre Carmen, José et Escamillo. Ils amplifient aussi l'admiration devant Carmen et devant Escamillo, ou encore, un chœur d'enfant caricature la relève de la garde. Toutes ces interventions nous poussent à ressentir de la sympathie pour Carmen, de l'admiration pour Escamillo, et de la pitié pour José.

Néanmoins, l'effet de contraste réaliste n'est pas seulement dû aux différentes formes musicales, mais aussi à la mise en scène par rapport à la situation spatiale de la trame. Au demeurant, même si elle est plutôt simplifiée par rapport à la nouvelle qui se déroule entre Cordoue, Séville, Gibraltar et les montagnes andalouses, l'opéra est tout de même divisé entre plusieurs lieux: le premier acte se déroule sur la place face à l'usine à tabac, le second dans la taverne de Lillas Pastia, le troisième dans les montagnes près de Séville, et le quatrième devant l'arène de la corrida. Tous les actes se situent dans la même ville, Séville, ou dans ses environs, mais le décor change absolument entre chaque localisation spatiale. Cela provient du soin que Bizet a pris de recréer la couleur locale donnée par Mérimée dans la nouvelle, faisant un tableau de la vie quotidienne espagnole dès la première scène: l'agitation de la place, les soldats puis les ouvrières qui fument sur scène, le jeu d'un triangle amoureux où les amants se passent discrètement un billet devant le mari trompé, à nouveau l'agitation

3 Cortés fait ici référence au "mélodrame" dans le sens musical du terme, c'est-à-dire une partie parlée sur un fond musical, face au dialogue parlé qui n'est pas accompagné de musique. 
dans la taverne puis à la corrida, etc. Cette couleur locale est renforcée par l'atmosphère transmise musicalement, tel que nous le décrit Cortés:

La creación de atmósferas es también notable. Los efectos orquestales tienen un sugestivo poder descriptivo o de evocación [...] En conjunto, los efectos orquestales que utiliza Bizet y el uso de motivos musicales crean un clima que siempre parece conducir a la tragedia. La atmósfera musical es sensual y voluptuosa, al tiempo que fatal; es a veces sofocante y parece propiciar inexorablemente las acciones, o bien orgiástica, como si las pasiones de los personajes impregnasen el entorno (Cortés, 1986: 31-32).

Effectivement, la musique transmet bien les sentiments et le symbolisme tout au long de l'opéra, mais l'utilisation de folklore espagnol est la ressource clé de l'exotisme et du dépaysement. Le compositeur fait preuve de sensibilité et de génie en reproduisant parfaitement la couleur locale du mythe sans avoir jamais visité le pays, et par-dessus tout, en utilisant ces formes de folklore, recours tout à fait inhabituel à cette époque dans l'opéra. Ainsi, certaines mélodies sont nées de l'inspiration de chansons espagnoles, mais seule "La Habanera" est le résultat d'une adaptation d'une chanson déjà existante. Nous considérons cette source d'inspiration comme une documentation sur la musicalité espagnole qui œuvre dans le but d'obtenir une couleur locale vraisemblable. Ceci n'est évidemment pas applicable à "La Habanera", mais nous opinons que cette adaptation a transformé une chanson folklorique banale en une mélodie enivrante -et l'une des plus célèbres au monde. Pour cela, Bizet a principalement changé le tempo de la chanson pour donner plus de fougue à sa mélodie. Nous pourrions dire qu'il s'agit là du génie du compositeur qui arrive à transmettre la couleur locale et le message de liberté bohémien sans en avoir jamais fait l'expérience empirique. D'autre part, au-delà des évidentes formes de flamenco et de chanson espagnole, Bizet introduit des instruments typiques, comme les castagnettes dont joue souvent Carmen, ou encore les bugles que sonnent les soldats. Par conséquent, nous avons là une autre innovation: sortir les instruments de musique de la fosse de l'orchestre pour les introduire sur scène. En définitive, nous observons à nouveau l'habilité de Bizet pour retransmettre la vraisemblance de la nouvelle de Mérimée, malgré la schématisation symbolique du récit et des personnages exigée par la forme opératique.

\subsection{La focalisation des narrations}

Nous en arrivons à un aspect plus épineux: la focalisation des deux récits. En nous basant sur la théorie de la narratologie de Gérard Genette dans Figures III, nous notons que Mérimée a décidé de jouer avec la narration en la faisant prendre en charge par deux personnages-narrateurs: un narrateur anonyme homodiégétique, l'archéologue, et un narrateur intradiégétique, don José. Sachant que cette nouvelle a tiré son inspiration d'un fait divers qu'il a appris de la bouche de son amie la Comtesse de Montijo, cela appuie la caractéris- 
tique de récit rapporté qu'il veut transmettre à la lectrice et au lecteur. Donnant ainsi une impression d'authenticité chère à cette époque en littérature. Ainsi, l'archéologue commence la narration pour faire l'exposition de la trame, et c'est don José qui prend la relève et lui raconte son histoire, devenant presque le narrateur principal et donnant une certaine distance dans la focalisation. Le narrateur-archéologue rapporte alors ce récit à la lectrice et au lecteur: “c'est de sa bouche que j'ai appris les tristes aventures qu'on va lire” (Mérimée, 2000: 67). À l'aide d'un flashback, José raconte son histoire depuis sa naissance: "Je suis né à Eliziondo, dit-il..." (Mérimée, 2000: 68). Tout ce processus narratif initial donne à la lectrice et au lecteur de nombreuses informations sur ce qui va se passer, leur donnant même connaissance de la fin tragique de l'histoire avant d'en connaître le début. Don José emploie dans sa narration de nombreux éléments qui rappellent qu'il n'est pas le narrateur principal, mais un narrateur intradiégétique. C'est le cas de l'apostrophe répétitive "Monsieur" ou des marques de modalités comme "Première sottise!" (Mérimée, 2000: 72). José commente ses sentiments et donne sans arrêt son point de vue sur les événements, et surtout sur l'attitude de Carmen, comme par exemple: "les Andalouses me faisaient peur; je n'étais pas encore fait à leurs manières: toujours à railler, jamais un mot de raison" (Mérimée, 2000: 70). Or, toute cette retranscription de l'histoire de José renforce l'impression de vraisemblance donnée par l'auteur, qui utilise un personnage-narrateur anonyme qui semble être son alter ego fictif. De cette façon, l'auteur fait presque croire à la lectrice et au lecteur qu'il s'agit d'une œuvre autobiographique sans avoir à y prétendre.

Quant à l'opéra, sa focalisation est conditionnée par le fait de voir la trame se dérouler en direct, ce qui élimine l'obligation de la présence d'un narrateur. En effet, bien qu'il soit possible d'inclure un personnage-narrateur homodiégétique, ce n'est pas habituel -tout du moins à cette époque. Ainsi, les librettistes ont supprimé le narrateur homodiégétique, reflet anonyme de Mérimée, et ont éludé la fonction de narrateur intradiégétique de don José. La représentation omnisciente de focalisation externe devient de cette façon plus vivante et pleine de vraisemblance, car le public a l'impression d'assister directement à la tragédie et c'est justement le style direct qui donne toute sa force à l'opéra. De plus, ils ont également laissé la surprise de la tragédie: à l'inverse de la nouvelle, le public ne s'attend pas à la mort de Carmen jusqu'à un certain point de l'opéra, et garde l'espoir d'une fin heureuse jusqu'à l'assassinat, et cela même si la fatalité plane tout au long de l'opéra. Cette narration objective laisse le soin au public de juger par lui-même les personnages, qui sont ainsi présentés objectivement, et non pas de suivre la tutelle d'un narrateur. Certes, la narration est en général beaucoup moins complexe, suivant l'histoire de façon linéaire, mais la narration omnisciente donne lieu à certains effets intéressants, tels que la surprise et l'implication du public.

D'autre part, suivant le schéma actantiel basé sur la classification d'éléments du récit en différents types d'actants de Greimas, celui-ci se constitue du sujet, José, et de l'objet, Carmen, dans l'axe du vouloir; et d'un autre côté, des opposants à la jonction entre le sujet 
et l'objet, comme Escamillo et Micaela dans l'hypertexte, ou comme les divers amants de Carmen dans l'hypotexte. Cependant, il semble manquer un actant adjuvant à cette relation conjointe, mais il existe un élément important et très certainement adjuvant: la fleur de cassie de Carmen qui envoûte don José et le fait tomber dans ses filets par un pouvoir de sorcellerie. Ce schéma actantiel est de même présent dans l'opéra, mais les librettistes ont décidé de créer ou fusionner certains opposants à la relation entre Carmen et José, pour des raisons pratiques telles que le nombres d'acteurs et l'équilibre des différents types de voix (par exemple, une présence féminine supplémentaire et opposée à la mezzo-soprano Carmen dans le personnage de la soprano Micaela), ainsi que pour donner de la force à la fatalité qui plane sur les personnages principaux. En effet, dans les deux textes, l'absence d'adjuvant en opposition à la présence de nombreux opposants reflète l'échec auquel la relation entre le sujet et l'objet est vouée.

\subsection{Micaela et le mal du pays}

Désormais, nous commencerons l'analyse des actants par l'opposant créé par les librettistes de l'opéra: Micaela. C'est avant tout un personnage qui illustre les valeurs chéries par José et par la société de l'époque: la vertu, la foi, la famille... Elle symbolise la campagne et la culture navarraises qui manquent tellement à don José: "je ne croyais pas qu'il y eût de jolies filles sans jupes bleues et sans natte tombant sur les épaules“ (Mérimée, 2000: 70), et le costume décrit par le livret de l'opéra est d'ailleurs fidèle à cette description. C'est un souvenir nostalgique de ses racines et de son passé, tout en étant douloureux: elle lui rappelle ce qu'il était et ce qu'il devrait être, elle le confronte à son échec et à ses faiblesses. Il s'agit d'une orpheline accueillie par la mère de don José, devenue donc la sœur de lait de celui-ci. Cependant, ils sont tout de même fiancés et il est fort possible qu'elle soit un choix de fiançailles d'origine œdipienne. De façon beaucoup plus évidente, il s'agit également de l'incarnation du rêve sédentaire et simple de don José, avant qu'il ne soit corrompu par Carmen. En effet, Micaela est issue du motif de l'épouse dévouée, le topo littéraire de la victime féminine face à la domination de l'homme: "Micaela permite recuperar con su simplicidad la tradición de las pálidas protagonistas románticas españolas [...] que acompañan, más en el sacrificio y en la renuncia que en el amor, a los héroes malditos de los dramas hispánicos" (González, 2007: 172). Elle s'oppose donc complètement au tempérament et au type de vie de Carmen. D'ailleurs, cette opposition est flagrante puisque Micaela apparaît pour la première fois dans son duo avec don José au début du premier acte, lorsqu'elle lui remet la lettre de sa mère, et cette scène se situe juste après la rencontre entre José et Carmen.

Ainsi, les librettistes juxtaposent les deux personnages féminins et Bizet intensifie leur opposition avec un air très différent de "La Habanera". En effet, les mélodies de Micaela ont un style beaucoup plus français: le compositeur n'utilise aucun élément de folklore espagnol, lais- 
sant de côté la passion langoureuse et le rythme frénétique et dansant de Carmen, pour favoriser une mélodie beaucoup plus traditionnelle, avec un tracé ample, linéaire et doux. C'est donc une élégance classique qui requière une voix pure et qui rappelle un peu les héroïnes de l'idéalisme pastoral baroque. L'orchestration, et surtout la harpe qui accompagne Micaela, suggère une ambiance romantique douce et traditionnelle, où les deux amants sont encore timides: la surprise et le silence interdit qui suivent l'échange des baisers nous touche de par cette innocence d'un amour pur et vacillant. Il est si fragile que l'esclandre provoqué par l'attaque de Carmen sur une ouvrière fait fuir Micaela et efface cet instant romantique et nostalgique de la scène.

L'amour de Micaela n'est pas moindre: il s'agit d'une forme de passion, plus posée et innocente que celle de Carmen, mais tout aussi imposante et surtout très émouvante. La musique de Micaela est forte et vibrante, comme les valeurs traditionnelles et le pays natal qu'elle symbolise. Cette puissance surprend par son opposition à l'aspect angélique et doux du personnage, souligné par le costume de Micaela,. Grâce à ce duo, Bizet réussit à introduire la nostalgie de don José: "Ma mère, je la vois, / je revois mon village! / Ô souvenirs d'autrefois! / doux souvenirs du pays!” (Halévy \& Meilhac, 1997: 27). Cette nostalgie est également présente dans la nouvelle, et nous fournit un symbole des valeurs traditionnelles et de l'innocence incarnées par ce personnage.

Enfin, 1'“Air de Micaela" ou "Je dis que rien ne m'épouvante" est le solo du personnage qui se trouve au milieu du troisième acte, lorsqu'elle cherche son fiancé entre les bandits, complètement terrifiée. Néanmoins, elle trouve la force dans son amour pour José pour se trouver dans ce milieu hostile, et surtout pour confronter la terrible Carmen, qui pourrait bien la tuer sans une hésitation, tout comme elle avait attaqué sa collègue de l'usine à tabac sous les yeux de Micaela. Elle trouve certainement cette force dans son amour, mais aussi dans sa foi religieuse, puisque, fidèle à son incarnation de la tradition, elle prie Dieu de la protéger et de lui insuffler du courage: "Vous me donnerez du courage, / vous me protégerez, Seigneur !" (Halévy \& Meilhac, 1997: 73). D'autre part, pour renforcer le caractère plus classique de ce solo, Bizet lui octroie le nom d'“air", alors que les autres solos ont des noms plus spécifiques et liés à la culture espagnole.

Par rapport à l'opposition entre Carmen et Micaela, elle se reflète dans celle qui existe entre José et Escamillo, même si elle ne représente aucune menace pour sa rivale, au contraire d'Escamillo. Ces deux opposants à la relation des héros sont à leur tour le reflet de l'opposition symbolique Nord-Sud, que forment déjà Carmen et José, comme pour renforcer ce contraste entre les deux cultures. Par conséquent, nous pouvons affirmer que les personnages sont basés sur un jeu d'oppositions, non seulement de caractères et de valeurs, mais aussi d'origines: ils représentent chacun un symbole qu'ils défendent jusqu'à la mort. Ce jeu d'oppositions a été, de plus, habilement renforcé par les librettistes grâce aux changements de personnages qu'ils ont faits. Nous pouvons aussi constater que la force du Sud et de la liberté l'emporte sur la tradition fermée du Nord. 


\subsection{Escamillo et la gloire du toréador}

Le second opposant à la relation entre les deux personnages principaux est évidemment Escamillo/Lucas, toréador et amant de Carmen. Dans la nouvelle, Lucas n'est toutefois pas celui qui occupe la place la plus importante entre les rivaux de José, mais il s'agit de la goutte d'eau qui fait déborder le vase. Cependant, il ne se confronte pas vraiment directement avec le héros. En effet, Mérimée a créé un autre personnage, García le Borgne, brigand et époux de Carmen. Celui-ci meurt en duel contre José, tout comme plusieurs autres de ses amants. Cependant, les librettistes ont préféré unir ces deux opposants en un seul, pour lui donner de la force: ils créent ainsi Escamillo, un toréador, comme Lucas, mais aussi le remplaçant de García le Borgne dans le duel face à don José. De cette manière, le livret offre un antagoniste beaucoup plus fort et plus présent que les multiples amants de la nouvelle, d'autant plus que ses apparitions en tant que remplaçant de Lucas sont beaucoup plus longues que dans la nouvelle. Mieux encore, sa figure de héros populaire est aussi intensifiée par rapport à celle de Lucas, créant une opposition beaucoup plus importante entre le toréador et don José, l'officier déserteur qui offre au contraire une figure d'antihéros. Il est d'ailleurs le symbole de ce que José pourrait être. Cette figure de héros populaire est surtout due à son succès en tant que toréador qui fait de lui le symbole de la virilité, ainsi que de la gloire. Bizet et ses librettistes en font un personnage presque principal, et c'est certainement parce qu'un tel héros ne peut pas être vaincu qu'ils ont éliminé l'allusion de Mérimée à un malencontreux accident lors d'une corrida, ou encore l'assassinat en duel face à José.

D'ailleurs, la chanson d'Escamillo, les "Couplets du Toréador" ou "Toréador, en garde !", est certainement la mélodie la plus connue de l'opéra, au même niveau que "La Habanera" de Carmen. Cette chanson apparaît au milieu du brouhaha de la taverne avec un caractère triomphal: d'abord annoncée par l'excitation des personnes présentes dans la taverne, la musique, appuyée par des trompettes et des percussions, est brillante et octroie un caractère arrogant et héroïque au personnage du toréador. D'ailleurs, Bizet indique "avec fatuité" dans la partition et fait réapparaître ce leitmotiv tout au long de l'opéra pour faire perdurer l'ambiance andalouse qu'elle transmet, même s'il la module de façon à transmettre d'autres sensations, telles que la menace, le triomphe ou la tragédie. Comme nous l'avons mentionné ci-dessus, les "Couplets du Toréador" tout comme Escamillo sont le symbole international de l'Espagne: la chanson se marie parfaitement avec le personnage, une mélodie triomphante et amusante, qui reste facilement en mémoire. Ces "Couplets du Toréador" sont cruciaux pour la couleur locale, car il s'agit de la mélodie qui caractérise le mieux l'Andalousie et surtout la tauromachie. À ce sujet, González nous donne l'appréciation suivante: 
La música con la que Bizet da cuenta de Escamillo tiene el efecto de una brillante vestimenta [...] Escamillo es más que un personaje porque tangencial con él vibra una atmósfera: la de la corrida [...] En el tema de la corrida está la clave de la capacidad evocadora de la obra [...] encontró la atmósfera múltiple y orgiástica acorde para ilustrar el escenario andaluz (González, 2007: 175).

En effet, les paroles de sa chanson font un tableau de la corrida, avec les acclamations de la foule et la description du combat avec le taureau:

Les spectateurs perdent la tête [...] Apostrophes, cris et tapage. / Poussés jusqu'à la fureur. / Car c'est la fête du courage [...] en combattant songe qu'un / œil noir te regarde / et que l'amour t'attend [...] Le taureau va... il vient... / il vient et frappe encore! [...] Le cirque est plein de sang (Halévy \& Meilhac, 1997: 47).

Escamillo se montre arrogant et prend plaisir à sa gloire. D'ailleurs, juste après la chanson, il prononce son amour envers Carmen qui est tout de suite séduite par ce héros, même si elle semble d'abord le rejeter, dans une tactique de séduction évidente. Cependant, la chanson se divise sur deux tons différents: l'un est menaçant, symbolisant le danger de la corrida et la tragédie à venir, et l'autre est joyeux et triomphal. Quant à son costume dans l'opéra, il s'agit de vêtements de señor espagnol, et surtout de toréador, normalement aux tons dorés, rougeoyants et noirs, symboles respectifs de la gloire, la fougue, le sang et la mort.

\subsection{Carmen et l'envô̂tement de la fleur de cassie}

Comme nous le savons tous, Carmen représente l'archétype de la femme fatale, qui remonte à l'Antiquité avec de grands mythes comme Lilith ou Salomé. Béatrice Grandordy définit le concept d'archétype de la femme fatale de la façon suivante:

C'est une femme [...] dont le comportement conscient ou inconscient vise à amener
l'homme à sa déchéance ou à sa perte ou à le placer dans une situation humiliante. La
Femme Fatale use habituellement de séduction et tend à user d'agressivité. [...] Enfin
il faut reconnaitre à la Femme Fatale un certain pouvoir. [...] Non seulement on ne
la manipule pas, mais de plus c'est elle qui obtient ce qu'elle veut, en premier lieu,
l'accomplissement de son destin. [...] La Femme Fatale n'est pas une meurtrière et ses
méfaits n'existent avant tout que dans la subjectivité d'autrui. [...] Elle veut qu'on lui
donne ce qu'elle refuse de donner en échange. (Grandordy, 2013: 13-14).

Carmen est certainement l'une des premières figures mythiques qui nous viennent à l'esprit en lisant cette description. Son pouvoir est celui de la séduction, en sorte de situation renversée du mythe de don Juan, et souffrant pourtant une réception totalement opposée. De fait, le motif du séducteur a été bien souvent acclamé pour sa grande habilité dans le libertinage et sa défense de la liberté individuelle, tandis que le motif de la femme 
fatale provoque une certaine terreur et même une certaine répulsion bien qu'elle présente exactement les mêmes caractéristiques. Cela démontre l'évidente situation patriarcale. Carmen est donc considérée comme une femme trop libre et frôlant même le motif de la sorcière -comme le montre Don José en la comparant au diable par exemple-. Les personnages masculins ressentent une grande inquiétude provoquée par cette indépendance et ce pouvoir féminins, de même que le ressentaient les spectatrices et les spectateurs qui ont critiqué le libertinage, ou plutôt les valeurs immorales du mythe. En effet, comme le souligne González:

La incomprensión que ha rodeado al personaje de Carmen reside en su radical polarización, en ese pasar de la más voluptuosa feminidad a la despótica y cruel ostentación de su libertad $[\ldots]$ habitualmente esa bipolaridad era encarnada por dos figuras femeninas antagónicas (González, 2007: 148).

Ces caractéristiques de femme fatale sont d'autant plus soulignées par la première apparition de Carmen dans la nouvelle de Mérimée, où elle surgit de la rivière telle une créature aquatique, faisant ainsi un net rapprochement entre Carmen et le mythe de la sirène, autre symbole du motif de la femme fatale et de sa séduction mortelle. De plus, les caractéristiques de la fatalité, de la sorcellerie et de la sensualité sont déjà indiquées implicitement par Mérimée dans le nom qu'il a choisi pour son personnage. Effectivement, le nom Carmen provient étymologiquement du latin carmina, qui veut dire "envoûtement" ou même "chant funèbre". Ceci combiné à l'utilisation très courante de ce nom en Espagne et à la jeune femme nommée Carmen rencontrée par Mérimée lors de ses voyages justifie d'autant plus le choix du nom du personnage. D'autre part, aucun personnage féminin ne lui fait de l'ombre: dans la nouvelle, il n'y en existe aucun, et dans l'opéra, autant ses compagnes bohémiennes, Frasquita et Mercedes, que Micaela sont complètement éclipsées par la présence explosive de cette personnalité excentrique.

D’autre part, comme nous l'avons signalé supra, Carmen représente une ethnie, la communauté gitane andalouse, et elle n'obtient pas son pouvoir de son statut social et économique -au contraire d'autre figures du motif de la femme fatale-, mais de sa seule personnalité envoûtante. Dans tous les cas, être bohémienne n'est pas un obstacle pour elle: au contraire, elle est fière d'être telle qu'elle est, de ses racines, de ses traditions et de ses croyances, mais avant tout de sa liberté, caractéristique principale de son ethnie. Carmen est la seule maîtresse de sa vie, dans le choix de ses amants, par exemple, mais aussi à l'heure de les délaisser. C'est toujours elle qui choisit qui sera son amant, même s'il ne s'intéresse pas à elle, comme dans le cas de José: ce désintérêt attise d'autant plus sa curiosité et pique son orgueil, et elle décide quand commencer la relation et quand y mettre fin. En relation avec ce pouvoir de décision, Dubois affirme: 
Conçue initialement par Mérimée comme un cas illustrant les traits d'une ethnie particulière, dans une contrée éloignée, en marge de la société, elle perd son caractère exotique et marginal pour prendre une place centrale dans la mythologie de la féminité, avec les caractères contradictoires qui s'attachent au complexe Eros / Thanatos. Elle incarne le danger des mésalliances et des femmes prodigues dans l'ordre familial traditionnel, la force dégradante de la fascination érotique, poussée jusqu'au maléfice et ne pouvant s'achever que par un drame et également l'énergie vitale, les forces de la joie, l'apothéose du plaisir sans mélange, une revendication de liberté poussée à l'absolu. Née du féminin singulier, elle perd sa singularité pour une image de la féminité conquérante dans la vie et lucide face à la mort, ivre de liberté et prise aux pièges de la destinée, universalisable en tous lieux géographiques et sociaux. C'est là ce qu'on peut appeler sa sublimation mythique. (Dubois in Brunel, 2002: 329)

De plus, et paradoxalement, c'est Carmen qui décide de se laisser tuer par José sans opposer aucun effort pour le faire changer d'avis, pour le calmer, ou encore, pour le séduire à nouveau. Avec le pouvoir qu'elle exerce sur lui, cela ne lui aurait rien coûté. Cependant, elle maintient sa liberté malgré tout, même si cela la mène vers la mort. D'ailleurs, dans la nouvelle elle déclare: "Comme mon rom, tu as le droit de tuer ta romi; mais Carmen sera toujours libre” (Mérimée, 2000: 110); tandis que dans l'opéra elle clame: "Jamais Carmen / ne cèdera, libre elle est née / et libre elle mourra" (Halévy \& Meilhac, 1997: 94). Ce sont les phrases décisives dans chaque œuvre pour déclencher l'assassinat final.

La liberté est le symbole le plus important représenté par la bohémienne, et elle devient par conséquent un symbole féministe, souvent utilisé pour la revendication de l'indépendance de la femme. ${ }^{4}$ Elle est surtout un symbole annonciateur d'un nouveau type de femme, au comportement libéré, qui exploite la sensualité féminine jusque dans ses plus grands extrêmes. Cependant, le côté fantastique qui lui attribue des pouvoirs de séduction surnaturelle justifie cette domination qu'elle a sur les hommes, chose qui pourrait être considérée comme un subterfuge pour faire accepter le personnage dans son contexte patriarcal où une telle femme est cause de scandale. Sa force et sa liberté lui confèrent une puissance presque frénétique dans sa façon d'aborder la vie, la faisant transgresser toute règle opposée à ses désirs sans aucune hésitation. Dans cette même ligne, Mérimée lui octroie un caractère presque félin, un côté sauvage qui se reflète surtout dans le regard noir de la jeune femme: “c'était une beauté étrange et sauvage [...] Ses yeux surtout avaient une expression à la fois voluptueuse et farouche" (Mérimée, 2000: 61). Quant au livret de l'opéra, il a adouci d'une certaine façon la personnalité de Carmen, et surtout ses méfaits: la plupart des crimes commis par la bande de brigands ont été supprimés, enlevant ainsi au personnage son statut d'instigatrice criminelle, et sa tendance à se prostituer n'y apparaît pas non plus, ne laissant place qu'à une simple frivolité de sa part.

4 La revendication féministe en est même arrivée à faire changer l'acte final de l'opéra dans la mise en scène de Leo Muscato (2018). Ici, Carmen n'est plus une victime: elle ne finit pas poignardée, au contraire, elle assassine elle-même son agresseur, Don José. Au sujet de l'utilisation féministe de ce mythe féminin, Catherine Clément montre parfaitement comment l'utiliser en leur faveur dans L'Opéra ou la Défaite des Femmes. 
En relation avec le côté surnaturel de ce mythe, tel que nous l'avons expliqué auparavant, il manque dans le schéma actantiel de Greimas un adjuvant à la jonction entre sujet et objet. Or, même s'il ne s'agit pas d'un actant, la fleur de cassie en est évidemment le principal adjuvant, puisque Carmen envoûte José au début de l'œuvre en lui jetant cette fleur qu'elle portait dans les cheveux. Après le chant gai des ouvrières et les supplications pressantes des hommes envers Carmen, celle-ci fait son apparition avec un changement abrupt de tempo, entonnant la mélodie de loin la plus connue de l'opéra et l'une des plus connues de la musique classique: "La Habanera" ou "L'amour est un oiseau rebelle". Le rythme rompt avec tout ce qui précède: il est typiquement flamenco, saccadé et syncopé, et donne au personnage un caractère langoureux, espiègle et séducteur. "La Habanera" englobe tout ce qui concerne le personnage de Carmen: son chant montre parfaitement ses atouts d'implacable séductrice, les paroles clament l'importance de la liberté pour la bohémienne, et l'accompagnement fait passer une impression de gravité menaçante, annonçant la tragédie liée au personnage dès sa première apparition: "La famosa 'Habanera' resume todo el talante erótico de Carmen [...] La melodía es sinuosa, provocativa [...] El acompañamiento, sin embargo, en ritmo de habanera, resulta terco y amenazador" (Cortés, 1986: 69). Or, la mélodie et l'accompagnement réapparaissent à tout moment dans l'opéra pour maintenir constamment cette sensation de fatalité latente. Les paroles suggèrent donc une forme d'amour libre qui est mis en rapprochement avec la liberté des bohémiens: "L'amour est un oiseau / rebelle que nul ne peut / apprivoiser, et c'est bien en vain qu'on l'appelle / s'il lui convient de refuser. [...] L'amour est enfant / de Bohême, il n'a jamais, / jamais connu de loi” (Halévy \& Meilhac, 1997: 15). Quant à la phrase tant répétée par Carmen et par le chœur, "Prends garde à toi !" (Halévy \& Meilhac, 1997: 15), elle montre à son tour le danger d'un tel amour. Le chœur d'hommes admiratifs et de femmes complices et envieuses amplifie le sentiment de danger face à cette séductrice capable de rendre un homme fou. Enfin, le moment où Carmen jette la fleur de cassie à don José est crucial, car il s'agit du moment où le public prend conscience de la présence dramatique de ce personnage masculin.

Bref, "La Habanera" est cruciale, mais Carmen chante d'autres airs devenus célèbres qui ont les mêmes caractéristiques musicales et symboliques que celui-ci. Ces deux solos de la gitane sont cette fois complètement créés par le compositeur français. Tout d'abord, nous avons la "Séguedille" ou "Près des remparts de Séville", qui conclut le premier acte avec l'envoûtement total de José, Carmen parvenant grâce à cette chanson à le convaincre de la laisser s'échapper de prison. Pour cela, elle lui promet en quelque sorte de l'aimer (“Je pense à certain officier, / qui m'aime, et qu'à mon tour, / je pourrais bien aimer!" (Halévy \& Meilhac, 1997: 39)) et fait de nouveau allusion à la liberté de l'amour ("Mon pauvre cœur / très consolable, / mon cœur est libre / comme l'air... / J'ai des amants à la douzaine, / mais ils ne sont pas à mon gré" (Halévy \& Meilhac, 1997: 38)). Ensuite, avec la "Chanson bohème" ou "Les tringles et les sistres tintaient", Carmen débute le second acte avec une danse fré- 
nétique, un rythme violemment syncopé, une voix qui s'épand dans le registre grave et qui nous semble transmettre une volonté de jouer avec le feu. Cette chanson est la culmination de l'agressivité et de la fougue de la bohémienne. D'autre part, ses paroles font une description lyrique des gitans:

Les anneaux de cuivre / et d'argent reluisaient / sur les peaux bistrées; / d'orange et de rouge zébrées / les étoffes flottaient au vent [...] Les Bohémiens à tour / de bras, de leurs instruments / faisaient rage, / et cet éblouissant tapage, / ensorcelait les zingaras! (Halévy \& Meilhac, 1997: 52).

Ceci renvoie immédiatement la lectrice et le lecteur de la nouvelle à son quatrième chapitre, où Mérimée décrit amplement les traits physiques et vestimentaires, ainsi que les coutumes festives des bohémiens. Donc, grâce aux solos de Carmen, Bizet nous offre une musique et les librettistes des paroles qui nous exposent tout le symbolisme de la bohémienne: sa liberté, son amour pour la vie, sa passion débordante, mais aussi ses origines tziganes.

Enfin, le costume andalou, jupe longue et corset à grand décolleté, qui est normalement utilisé pour caractériser le personnage est crucial: généralement de tons rougeoyants et noir, il symbolise la passion et la mort qu'elle incarne. D'ailleurs, il est commun de voir une évolution du costume tout au long de l'opéra, commençant normalement par des tons plus clairs mêlés au rouge, pour devenir de plus en plus obscurs et finissant souvent par un costume presque totalement noir pour le dernier acte -avec une touche de rouge habituelle, comme la fleur de cassie prise dans les cheveux, ou les lèvres vermeilles. D'autre part, certains metteurs en scène se plaisent à montrer le personnage en train de se laver (comme Francesca Zambello dans sa mise en scène de 2007 au Royal Opera House, ou Richard Eyre en 2009 au Metropolitan Opera), faisant ainsi allusion à son apparition dans la nouvelle telle une créature aquatique, en plus du côté érotique, provocateur et intimiste que cela implique.

\subsection{Don José et la fatalité latente}

Quant à don José, c'est un personnage principal qui s'oppose totalement à la force et à la liberté de Carmen: il tombe généralement dans le domaine du pathétisme, incapable de dominer ses passions malgré sa volonté d'accomplir son devoir. Cette lutte intérieure le ronge de telle façon qu'il devient colérique et possessif. Il ne peut pas accepter d'avoir perdu son honneur pour rien et, aux dires de González, il développe une "mala conciencia que lo obligará no sólo a sentirse culpable, sino también a responsabilizar de su supuesta degradación a la mujer que simboliza ese tipo de vida, los valores ante los cuales sucumbió por fatal atracción" (González, 2007: 167). De plus, le fait qu'elle ne se sente absolument pas coupable, étant donné qu'elle ne lui a jamais demandé de tout sacrifier pour elle, le 
confronte à sa médiocrité et ne fait qu'amplifier sa frustration. Pourtant, il essaie de la faire sentir coupable et de l'obliger à lui rester fidèle et à l'aimer en utilisant cet argument dans une tentative désespérée de chantage émotionnel: en échange de ses sacrifices, il exige d'elle un sacrifice en retour, celui de sa liberté, alors qu'il s'agit paradoxalement et inconsciemment de ce qui l'attire le plus chez elle; en effet, il voudrait la convertir en Micaela, alors qu'il l'a abandonnée à cause de l'ennui qu'elle lui cause en comparaison avec la personnalité fougueuse de Carmen. Il ne peut rien faire face à la séduction de cette femme fatale qui le domine totalement, transformant le stéréotype masculin de dominateur en impuissante victime. D'ailleurs, dès leur rencontre, il est tout de suite attiré et séduit, mais il est en même temps effrayé du pouvoir qu'elle détient sur lui. Dans l'opéra, il déclame: “Certainement s'il y a / des sorcières, cette fille-là / en est une" (Halévy \& Meilhac, 1997: 19). Finalement, il est presque obligé de l'assassiner pour tuer ce qu'il considère sa faiblesse, pour se libérer de sa mauvaise conscience: le meurtre est la seule issue à ce combat intérieur entre passion et honneur.

Néanmoins, don José est un personnage complet au début du récit, avant que sa personnalité ne s'efface peu à peu à cause de son envoûtement et de son obsession pour Carmen. C'est un homme simple et honnête: son rêve est de monter en grade en tant que militaire et de fonder un foyer avec une jeune fille de son pays, c'est-à-dire qu'il désire avoir une vie sans complication. Amoureux des traditions, nous pourrions dire qu'il est presque obsédé par les conventions sociales et les devoirs d'un militaire: du respect de ces règles dépend l'intégrité de son honneur, ce qui est crucial pour lui. Ceci fait en sorte qu'il ait un esprit très fermé à toutes sortes de transgressions, ce qui l'empêche par exemple de s'ouvrir à une relation libre avec Carmen. D'autre part, sa sédentarité naturelle le rend constamment nostalgique, surtout quand il devient un bandit fugitif. D'ailleurs, nous pouvons affirmer qu'il représente le Nord de l'Espagne, ce qui l'oppose à Carmen, symbole de l'Andalousie, région dont les mœurs sont bien trop fougueuses pour lui. Effectivement, la famille, la religion et son pays sont certainement les trois valeurs les plus importantes pour lui: il est très fier de ses origines. Dans la nouvelle, il s'introduit au narrateur de la façon suivante: “Je m'appelle don José Lizarrabengoa [...] je suis Basque et vieux chrétien. Si je prends le don, c'est que j'en ai le droit” (Mérimée, 2000: 68). De même, dans l'opéra, il dit à Zúñiga: “[José] c'est la coutume de la Navarre... / ça me rappelle le pays... / [Zúñiga] Vous êtes Navarrais? / [José] Et vieux chrétien. / Don José Lizarrabengoa, / c’est mon nom..." (Halévy \& Meilhac, 1997: 12). Il ajoute ensuite qu'il était destiné à devenir homme d'église, mais qu'il a dû s'enrôler après une dispute pour sauver son honneur au jeu de paume, information qui nous donne une première idée de sa tendance à s'attirer des ennuis et à recourir à la violence pour les résoudre.

Par rapport à la fatalité latente qui l'accompagne, elle est particulièrement présente dans deux scènes qui annoncent clairement la tragédie finale: l'une d'entre elles est la "Ro- 
mance de la fleur" ou "La fleur que tu m'avais jetée", chantée par José dans son duo avec Carmen à la fin du second acte. En effet, les premières traces de la discorde apparaissent, puisque les deux personnages se disputent et se quittent froidement. Le duo est composé de supplications de José qui sort la fleur de cassie séchée pour démontrer son amour, et d'un fredonnement capricieux de Carmen, qui joue des castagnettes et danse de façon dédaigneuse face au dilemme de José, qui est déchiré entre l'appel des trompettes de la garde et l'attrait de la fuite avec Carmen, une claire représentation de ce combat entre passion et honneur. Blas Cortés nous signale à ce sujet: "Este insólito y original contrapunto en la historia de la ópera -castañuelas y trompetas- plantea dramáticamente la dialéctica entre ambos mundos [...] resulta tenso y glacial" (Cortés, 1986: 121). Dans cette romance, le pathétisme de don José apparaît pour la première fois avec autant de force: "Je me prenais à te maudire / à te détester, à me dire: / Pourquoi faut-il que le destin / L'ait mise sur mon chemin [...] Carmen, je t'aime!"(Halévy \& Meilhac, 1997: 55). Il montre son désespoir qui vire à l'angoisse face au détachement de Carmen qui s'oppose à un amour si dépendant. Cette déclaration d'amour est accompagnée par un ton menaçant et grave qui symbolise à la fois le début de la discorde et le destin tragique qui les attend.

Toutefois, c'est surtout le "Trio de Cartes" du troisième acte qui ouvre définitivement la porte à la tragédie. Les librettistes ont réutilisé l'élément de la nouvelle, le tarot, fantastique et très présent dans la culture bohémienne, pour révéler de façon définitive à Carmen sa mort et celle de don José: "la mort! / J'ai bien lu... moi d'abord. / Ensuite lui... pour tous / les deux la mort" (Halévy \& Meilhac, 1997: 64). La sentence donnée par Carmen sur un ton grave et menaçant débute la croissance de la tension, jusqu'à trouver son apothéose dans l'assassinat final. Ses amies, Frasquita et Mercedes, qui ont assisté à la révélation des cartes, l'avertissent du retour de José, mais, fière, elle répond: "Je ne suis pas femme / à trembler devant lui..." (Halévy \& Meilhac, 1997: 88). José revient après son départ menaçant du troisième acte pour supplier son pardon: "Je ne menace pas, / j'implore, je supplie [...] Carmen, il est temps / encore" (Halévy \& Meilhac, 1997: 90). Ce à quoi elle réplique, inflexible et courageuse: "Non, je sais bien / que c'est l'heure, je sais bien / que tu me tueras, / Mais que je vive ou que je / meure, non! non!non! / Je ne céderai pas” (Halévy \& Meilhac, 1997: 90).

C'est surtout lorsqu'elle dit ne plus l'aimer que José entame sa prière la plus passionnée, dans son désespoir d'obtenir un amour en retour du sien. C'est alors que la phrase inspirée de la nouvelle est utilisée: "Jamais Carmen / ne cédera, libre elle est née / et libre elle mourra" (Halévy \& Meilhac, 1997: 94). La dispute entre les deux personnages est d'un dramatisme prenant, qui est mis en relief par la simultanéité entre la corrida, représentée en fond sonore par les "Couplets du Toréador", et l'assassinat en premier plan de Carmen par José. Cette mort est nécessaire -d'où l'insistance de Bizet à la représenter-, comme le souligne Dubois: 
La mort de Carmen est perçue comme un acte de purification de la part de la société qui se débarrasse d'un monstre. C'est là une récupération de la fantasmagorie éternelle liée à la peur de l'autre sexe, mais particulièrement active depuis la fin du XIX ${ }^{\mathrm{e}}$ siècle. (Dubois in Brunel, 2002: 332)

Cette originalité qui met en parallèle les deux morts héroïques et artistiques donne une esthétique qui montre le génie de Bizet et qui nous mène à la catharsis finale. Le chœur interne chante donc la chanson d'Escamillo, décrivant la corrida qui correspond à l'altercation et à la scène sanglante que nous voyons en premier plan entre Carmen et José. Les coups de poignards sont ensuite accompagnés d'accord stridents et forts, suivis par les vivats de la foule qui acclame la victoire d'Escamillo dans l'arène, et qui semble ainsi acclamer l'assassinat de Carmen. Cette scène finale est la culmination de toutes les caractéristiques de l'opéra: le destin tragique se résout sur un mélange de genres et de thèmes, avec la gloire du toréador, le pathétisme de José, la liberté inconditionnelle de Carmen, l'amour, la mort, la tauromachie... tout le symbolisme si cher à Mérimée.

\section{Conclusion}

La transmodalisation à l'opéra de Bizet, Meilhac et Halévy de la nouvelle de Mérimée est un chef-d'œuvre qui a servi à remettre en valeur l'œuvre originale de par son grand succès. En effet, la nouvelle, comme la plupart des écrits de Mérimée, a été critiquée comme trop sèche dans la description des sentiments et trop pédante dans ses commentaires sur l'archéologie ou la linguistique pour intéresser pleinement les lecteurs. La focalisation complexe divise la trame en deux parties à travers le jeu des deux narrateurs. Cet effet de vraisemblance est certes intéressant, mais il rompt également la continuité et l'unité de la trame. Bref, il s'agit d'une lecture plaisante, mais qui n'a pas reçu le succès qui annoncerait sa postérieure mythification.

D’un autre côté, l'opéra offre des mélodies qui plaisent et ne vieillissent jamais, comme le démontre son énorme succès international depuis sa création jusqu'à l'actualité, et qui a même fini par gagner le public espagnol, d'abord réticent face à cette représentation de sa culture. Le scénario est excellent et plein d'émotions grâce à l'adaptation judicieuse de la trame et de la focalisation de la nouvelle, grâce à une partition brillante, intense et passionnée, à une abondance de personnages bien définis et à une couleur locale resplendissante. Même si celle-ci provient d'un stéréotype de l'Espagne et qu'elle le promeut, il s'agit d'une image universelle qui est parvenue à s'implanter dans ce pays qui l'a d'abord rejetée. Les librettistes et le compositeur ont donc fait un grand travail de compréhension et d'interprétation de l'œuvre de Mérimée et ont pu saisir ainsi les aspects les plus importants pour l'écrivain, comme la linguistique, la tauromachie, la couleur locale, la cruelle réalité, les motifs de la femme fatale et de la femme dévouée, celui de l'antihéros, l'opposition entre le Sud et le 
Nord de l'Espagne, la superstition, la violence et la fatalité. Il s'agit donc d'un opéra unique en son genre et en son siècle, puisque, malgré l'adoucissement des mœurs effectué par les librettistes, il est le seul de l'époque à montrer une réalité aussi cruelle.

\section{Références bibliographiques}

Albouy, Pierre. 1998. Mythes et mythologies dans la littérature française. Paris, Armand Colin.

BACKès, Jean-Louis. 1994. Musique et Littérature. Essai de poétique comparée. Paris, PUF.

Cortes, Blas. 1986. “Carmen” de Georges Bizet. Madrid, Daimon (coll. Introducción al mundo de la ópera).

Dubors, Claude-Gilbert. "Carmen” in Brunel, Pierre. 2002. Dictionnaire des mythes féminins. Paris, Éditions du Rocher.

Genette, Gérard. 1972. Figures III. Paris, Seuil (coll. Poétique).

Genette, Gérard. 1982. Palimpsestes. La Littérature au second degré. Paris, Seuil (coll. Poétique).

González Troyano, Alberto. 2007. “Don Juan”, “Fígaro”, “Carmen”. Sevilla, Fundación José Manuel Lara.

GRANDORDy, Béatrice. 2013. La Femme Fatale: ses origines et sa parentèle dans la modernité. Paris, L'Harmattan.

Halevy, Ludovic \& Henry Meilhac. 1997. Carmen: Opéra comique en quatre actes. Illustration Hippolyte Romain. Paris, Gallimard (coll. Joëlle Losfield).

Herrero, Fernando. 1983. La ópera y su estética (para una práctica escénica). Madrid, Dirección General de Música y Teatro - Ministerio de Cultura.

JACOBS, Arthur \& Stanley SADIE. 1998. El libro de la ópera. Madrid, Rialp.

Lefter, Diana-Adriana. 2008. Introduction à l'étude du mythe dans la littérature. Bucarest, Editura Universitãtii din Bucuresti.

LeOn, Paul. 1962. Mérimée et son temps. Paris, PUF.

MaY, Robin. 1987. Guía de la ópera. Barcelona, Plaza \& Janés.

MÉrimée, Prosper. 2000. Carmen. Paris, Gallimard (coll. Folio Classique).

Pujante, David. 2012. "La ópera: espectáculo y texto literario" in Alemany Ferrer, Rafael \& Francisco CHIco Rico (éds.). Literatura y espectáculo. Alicante, Publicaciones de la Universidad de Alicante.

Rouart, Marie-France. "La Tsigane" in Brunel, Pierre. 2002. Dictionnaire des mythes féminins. Paris, Éditions du Rocher. 
Anales de Filología Francesa, . $^{\circ}$ 28, 2020

APPROCHE NARRATOLOGIQUE À LA TRANSMODALISATION DE CARMEN: DU RÉCIT LITTÉRAIRE À...

Ruwet, Nicolas. 1972. Langage, musique, poésie. Paris, Seuil.

San Miguel, Manuela. 1984. Mérimée. Erudición y creación literaria. Salamanca, Publicaciones de la Universidad de Salamanca. 